\title{
Indications to surgery in necrotising enterocolitis of extremely low birth weight prematures. can we do better?
}

Volume 6 Issue 2 - 2017

\section{Introduction}

\section{A Disease of the premature}

Low birth weight prematures are affected by multiple function immaturity that influence survival and outcome. Although increased understanding of physiology and technology advancements have decreased mortality, still survival is directly related to birth weight.

Extremely low birth weight infant (ELBW) $(<1000$ grams) remain at high risk for death and disability with $30-50 \%$ mortality and at least $20-50 \%$ risk of morbidity in survivors.

Survival of these high risk patients has been reported to be $20 \%$ when birth weight is between 500 and $600 \mathrm{~g}$, with a $50 \%$ incidence of significant neurodevelopmental impairment in $500-750 \mathrm{~g}$ infants ${ }^{1}$ Due to this weight related risk, some authors even defined as incredibly low birth weight (ILBW) a subset of patients whose birth weight is less than $750 \mathrm{gm}$, although a systematic characterization of such a group is lacking. ${ }^{2,3}$

Necrotising enterocolitis is one of the major complications affecting these patients, often leading to an unfavorable outcome despite surgical treatment, because of intestinal necrosis and sepsis. Early stages of NEC are treated conservatively, but understanding progression of the disease and optimal timing of surgery is often challenging.

The indications for surgery include the presence of pneumoperitoneum, indicating perforation of the intestine, clinical deterioration despite maximal medical treatment, abdominal mass with intestinal obstruction, and development of intestinal stricture. Relative indications include fixed dilated intestinal loop, presence of portal gas, thrombocytopenia, and rapid fall in platelet count. ${ }^{4,5}$

Although relative indications to surgery may apply, the absolute indication to surgery in prematures with NEC is Bell stage III, which is characterized by the aforementioned clinical features of advanced disease and progressive deterioration. This condition involves high fatality. Patients who undergo surgery are more likely to die than the ones who do not. ${ }^{6}$

Gangrene and perforation are known to be associated with $30 \%$ and $64 \%$ postoperative mortality rate respectively ${ }^{7}$ and the outcome of affected infants is influenced by the length of necrotic bowel. ${ }^{8}$

\section{The impact of surgery}

Surgery most commonly consists of laparotomy, resection of the necrotic bowel and ileostomy. Different surgical techniques such as diverting jejunostomy or 'clip and drop' have been described to deal with extensive disease and avoid massive small bowel resection,

\author{
Andrea Zangari,' Caterina Gulia, ${ }^{2}$ Roberto \\ Piergentili ${ }^{3}$ \\ 'Azienda Ospedaliera San Camillo Forlanini, Italy \\ 2Universita degli Studi di Roma La Sapienza, Dipartimento di \\ Urologia, Italy \\ ${ }^{3}$ Institute of Molecular Biology and Pathology, Italian National \\ Research Council (CNR-IBPM), Italy
}

Correspondence: Andrea Zangari,Azienda Ospedaliera San Camillo Forlanini, Roma, Italy, piazza Carlo Forlanini, 4, Roma, Italy, Tel 393000000000, Fax 391000000000,

Email andreazangari@libero.it

Received: January 30, 2016 | Published: February 07, 2017

while survival seems not to be affected by laparotomy compared to peritoneal drainage. The advantage of resection and primary anastomosis over stoma formation is still controversial. ${ }^{9}$ Although prospective studies and randomized controlled trials are needed to define the best operative treatment for neonates with severe NEC, resection with ileostomy is the most frequently used procedure. ${ }^{9-11}$

Enterostomy has always played a consistent role in the surgical treatment of NEC, with the purpose of decompression and protection of questionable bowel from further damage. ${ }^{12}$ Yet it is usually performed in association with resection of necrotic bowel when current indications for surgery are encountered, that is in advanced stages of the disease. Although it has rarely been investigated for potential benefits as an isolated procedure, it resulted in statistically significant improvement of survival, especially in low- and extremely low birth weight patients ${ }^{13,14}$ and it has been shown to prevent progression of disease at earlier stages. ${ }^{15-17}$

Extremely low birth-weight (ELBW), premature infants who develop necrotizing enterocolitis (NEC) or isolated intestinal perforation (IP) represent a group of patients with extremely high morbidity and mortality. ELBW refers specifically to infants with birth weight $<1000 \mathrm{~g}$. Multiple case series report the mortality of ELBW infants that undergo operation for NEC to be approximately $50 \%{ }^{11,18,19}$

In VLBW infants with NEC, surgery has not been found to directly correlate with mortality, yet this was associated with extensive intestinal involvement and/or advanced disease. In an appropriate setting, operative intervention under general anesthesia has been 
shown to be well tolerated by VLBW infants with NEC, independently from birth weight and G.A. ${ }^{20}$

\section{Earlier indications to surgery?}

Due to anaesthesiological and surgical risk concerns, standard indications are usually delayed to the life-threatening condition of advanced disease, which includes perforation and clinical deterioration. A more timely surgical intervention may be defined by panels describing the trajectory of metabolic derangement. ${ }^{21}$ Nonetheless, according to some authors, early primary laparotomy is safe and effective in ELBW infants and survival seems to be affected by the length of necrotic bowel rather than by surgery itself or by age and weight at surgery. ${ }^{8,22}$

While novel forms of treatment, including whole-body moderately controlled hypothermia and administration of amniotic fluid stem cells, are currently under investigation, ${ }^{23}$ the surgeon is facing with increasingly fragile patients who survive premature birth. In the light of the previous considerations, new attention should be focused on the possible role of surgery in preventive measures, in high risk selected cases, in which waiting for free air may be too late. ${ }^{21}$ Although standard indications to surgery for NEC still rely on signs of advanced disease, it can be reasonably hypothesized that in selected extremely challenging situations as ELBW or ILBW, laparotomy and enterostomy at an earlier stage than usual may be worth of new consideration, in order to prevent further progression of disease, which seems to directly correlate with mortality rather than surgery itself. New randomized studies on this challenging matter are warranted to achieve the basis of a new perspective of current surgical indications for NEC.

\section{Acknowledgments}

None.

\section{Conflicts of interest}

Author declares there are no conflicts of interest.

\section{Funding}

None.

\section{References}

1. Stoll BJ, Chapman IA. The High-Risk Infant. In: Kliegman, Behrman, Jenson, Stanton editors. Nelson Text book of Pediatrics. (18th edn), Saunders Elsevier, Philadelphia USA. 2007.

2. Naggar ME. Pediatric Clinical Diagnosis. (6th edn), Jaypee Brothers Medical Publishers, New Delhi, India. 2008. P.24.

3. Rahman AFMM, Solanky J, Mridha MA, et al. Lowest Birth Weight Baby Survived in Abha General Hospital, Abha, K S A. Bangladesh J Child Health. 2011;35(2):71-74.

4. Pierro A. The surgical management of necrotising enterocolitis. Early Hum Dev. 2005;81(1):79-85.

5. Patel BK, Shah JS. Necrotizing Enterocolitis in Very Low Birth Weight Infants: A Systemic Review. ISRN Gastroenterol . 2012;2012:562594.
6. Bălănescu RN, Topor L, Drăgan GC. Clinical and surgical aspects in necrotizing enterocolitis. Chirurgia (Bucur). 2013;108(2):184-188.

7. Kosloske AM. Surgery of necrotizing enterocolitis. World Journal of Surgery. 1985;9(2):277-284.

8. Gfroerer S, Fiegel H, Schloesser RL, et al. Primary laparotomy is effective and safe in the treatment of necrotizing enterocolitis. World $J$ Surg. 2014;38(10):2730-2734.

9. Rees C, Hall N, Eaton S, et al. Surgical strategies for necrotising enterocolitis: a survey of practice in the United Kingdom. Arch Dis Child Fetal Neonatal Ed. 2005;90(2):F152-F155.

10. Pierro A, Hall N. Surgical treatment of infants with necrotizing Enterocolitis. Semin Neonatol. 2003;8(3):223-232.

11. Blakely ML, Lally KP, McDonald S, et al. Postoperative Outcomes of Extremely Low Birth-Weight Infants With Necrotizing Enterocolitis or Isolated Intestinal Perforation: A Prospective Cohort Study by the NICHD Neonatal Research Network. Ann Surg. 2005;241(6):984-994.

12. Lin PW, Stoll BJ. Necrotising enterocolitis. Lancet. 2006;368(9543):1271-1283.

13. Błaszczyński M, Porzucek W, Becela P, et al. T-tube enterostomy in the surgical treatment of necrotizing enterocolitis. J Ped Neonatal. 2005;2(4):79-83.

14. Rygl M, Pycha K, Stranak Z, et al. T-tube ileostomy for intestinal perforation in extremely low birth weight neonates. Pediatr Surg Int . 2007;23(7):685-688.

15. Martin LW, Neblett WW. Early operation with intestinal diversion for necrotizing enterocolitis. J Pediatr Surg. 1981;16(3):252-255.

16. Saleem MM, Skef Z, Bashir O, et al. Necrotizing enterocolitis, surgical experience. Saudi J Gastroenterol. 1997;3(1):41-45.

17. Ferrara F, Angotti R, Burgio A, et al. Ileostomy in extremely low birth weight and premature neonates. Minerva Pediatr. 2013;65(4):411-415.

18. Ricketts RR, Jerles ML. Neonatal necrotizing enterocolitis: experience with 100 consecutive surgical patients. World J Surg . 1990;14(5):600-605.

19. Ketzer de Souza JC, da Motta UIC, Ketzer CR. Prognostic factors of mortality in newborns with necrotizing enterocolitis submitted to exploratory laparotomy. J Pediatr Surg . 2001;36(3):482-486.

20. Anveden-Hertzberg L, Gauderer MW. Surgery is safe in very low birthweight infants with necrotizing enterocolitis. Acta Paediatr. 2000;89(2):242-245.

21. Tepas JJ, Leaphart CL, Plumley D, et al. Trajectory of Metabolic Derangement in Infants with Necrotizing Enterocolitis Should Drive Timing and Technique of Surgical Intervention. J Am Coll Surg. 2010;210(5): 847-854

22. Alexander F, Smith A. Mortality in micro-premature infants with necrotizing enterocolitis treated by primary laparotomy is independent of gestational age and birth weight. Pediatr Surg Int . 2008;24(4):415-419.

23. Hall NJ, Eaton S, Pierro A. Royal Australasia of Surgeons Guest Lecture. Necrotizing enterocolitis: prevention, treatment, and outcome. J Pediatr Surg. 2013;48(12):2359-2367. 